\title{
Clonal Expansion of New Penicillin-Resistant Clade of Neisseria meningitidis Serogroup W Clonal Complex 11, Australia
}

\author{
Shakeel Mowlaboccus, Keith A. Jolley, \\ James E. Bray, Stanley Pang, Yung Thin Lee, \\ Jane D. Bew, David J. Speers, Anthony D. Keil, \\ Geoffrey W. Coombs, Charlene M. Kahler
}

In Western Australia, Neisseria meningitidis serogroup W clonal complex 11 became the predominant cause of invasive meningococcal disease in 2016. We used core-genome analysis to show emergence of a penicillin-resistant clade that had the penA_253 allele. This new penicillin-resistant clade might affect treatment regimens for this disease.

$I^{\prime}$ nvasive meningococcal disease (IMD) is caused by a meningococcus, Neisseria meningitidis. The main manifestations of this disease are septicemia or meningitis. Meningococcal strains can be classified into 12 serogroups phenotypically and into sequence types (STs) by multilocus sequence typing (1). Similar STs are grouped into the same clonal complex (cc). IMD is most commonly caused by isolates expressing a serogroup A, B, C, W, X, or Y polysaccharide capsule. Until recently, serogroup A was the major cause of disease in Africa (2). Serogroups B, C, and Y continue to predominate in the United States, Europe, Asia, and Australia $(3,4)$.

In Australia, after introduction of serogroup $\mathrm{C}$ conjugate vaccine in the national immunization program in 2003, incidence of serogroup $\mathrm{C}$ has decreased; serogroup B predominated during 2004-2015. However, during 2016, the prevalence of serogroup $\mathrm{W}$ disease increased because of $N$. meningitidis strains in the cc11 lineage (MenW:cc11) $(5,6)$, which have also been reported worldwide. Although extensive core-genome analyses of these MenW:cc11 strains have been reported $(7,8)$, antimicrobial drug susceptibility of these clinical isolates has not been generally reported.

Author affiliations: University of Western Australia, Perth, Western Australia, Australia (S. Mowlaboccus, D.J. Speers, C.M. Kahler); University of Oxford, Oxford, UK (K.A. Jolley, J.E. Bray); Murdoch University, Murdoch, Western Australia, Australia (S. Pang, Y.T. Lee, G.W. Coombs); Fiona Stanley Hospital, Perth (S. Pang, G.W. Coombs); Queen Elizabeth II Medical Centre, Perth (J.D. Bew, D.J. Speers); Princess Margaret Hospital for Children, Perth (A.D. Keil); Telethon Kids Institute, Perth (C.M. Kahler)

DOI: https://doi.org/10.3201/eid2308.170259
Although penicillin has been used for control of IMD, clinical isolates relatively resistant to this drug have been reported worldwide. For meningococci, a penicillin MIC $>2 \mathrm{mg} / \mathrm{L}$ is caused by plasmid-mediated $\beta$-lactamase production but is extremely rare (9). Conversely, isolates conferring intermediate resistance to penicillin (MIC $0.12-0.25 \mathrm{mg} / \mathrm{L}$ ) are uncommon but the frequency of these isolates varies geographically. The mechanism of relative resistance in these isolates involves expression of altered forms of 1 of 4 penicillin-binding proteins (PBPs) that are involved in peptidoglycan biosynthesis during bacterial growth and cell division (10).

Although treatment with penicillin is still effective against these penicillin-intermediate strains, low-dose treatment regimens may fail for cases involving penicillinresistant isolates (MIC $\geq 0.5 \mathrm{mg} / \mathrm{L}$ ) (11). We report recent emergence and clonal expansion of a phylogenetically related cluster of penicillin-resistant MenW:cc11 isolates in Western Australia.

\section{The Study}

Western Australia is the largest state in Australia (land area 1.02 million square miles). However, it has a population of only 2.5 million persons. In concordance with the national trend, there has been a shift in the predominant serogroup in Western Australia; MenW was responsible for most IMD cases in 2016. The first laboratory-confirmed MenW:cc11 case in Western Australia was recorded in April 2013 and was the only MenW case for that year. Since that time, an additional $18 \mathrm{MenW}$ :cc11 laboratory-confirmed cases have been reported, representing $11 \%(\mathrm{n}=2)$ of all IMD cases in $2014,27 \%(\mathrm{n}=3)$ in 2015 , and $67 \%(\mathrm{n}=13)$ in 2016, a significant increase from 2014 through 2016 (p = 0.0004 , by Fisher exact test). Three deaths were caused by MenW:cc11 infection, 1 in 2015 and 2 in 2016.

The 19 MenW:cc11 strains isolated during January 1, 2013-December 31, 2016, were assessed for susceptibility to penicillin, ciprofloxacin, ceftriaxone, and rifampin. We performed drug susceptibility testing by using the Etest (bioMérieux, Marcy l'Etoile, France). MIC results were interpreted according to Clinical Laboratory Standard Institute (http://clsi.org) breakpoints. All isolates were susceptible to ciprofloxacin (MIC $\leq 0.03 \mathrm{mg} / \mathrm{L}$ ), ceftriaxone $(\leq 0.12$ $\mathrm{mg} / \mathrm{L})$, and rifampin $(\leq 0.5 \mathrm{mg} / \mathrm{L})$. However, variation in 
penicillin susceptibility was observed: 8 were susceptible $(\leq 0.06 \mathrm{mg} / \mathrm{L}), 2$ were less susceptible $(0.12-0.25 \mathrm{mg} / \mathrm{L})$, and 9 were resistant $(\geq 0.5 \mathrm{mg} / \mathrm{L})$. All isolates less susceptible to or resistant to penicillin were identified in 2016.

We further characterized isolates by using whole-genome sequencing with the Miseq Platform (Illumina, San Diego, CA, USA). Raw reads were assembled, auto-tagged, and curated by using the BIGSdb genomics platform on the PubMLST website (http://pubmlst.org/neisseria) (12). Four STs, all belonging to cc 11 , were identified: ST-11 $(\mathrm{n}=11)$, ST-1287 $(\mathrm{n}=2)$, ST-3298 $(\mathrm{n}=1)$, and ST-12351 $(\mathrm{n}=5)$. All isolates had the same PorA:FetA profile (P1.5,2:F1-1) as that identified in the MenW:cc11 collection responsible for outbreaks in South America and the United Kingdom (8). Furthermore, genomic sequences indicated the isolates from Western Australia were within the same United Kingdom-South America cluster as isolates from the eastern coast of Australia (13).

Phylogenetic analysis of the meningococcal core genome (14) identified 2 distinct clusters within the MenW:cc11 population of Western Australia (Figure 1). One isolate (ExNm672) was an outlier and could not be clustered. ExNm672 was isolated from a traveler from
Asia who had recently arrived in Western Australia, which would likely explain the different genealogy of this strain. All isolates less susceptible to or resistant to penicillin were in cluster B. Geocoding analysis showed that the 10 isolates in cluster B were obtained from 7 geographically well-separated regions in Western Australia. This observation suggests successful expansion of a new penicillinresistant clone in 2016.

For $N$. meningitidis, polymorphisms within the gene encoding PBP2, also known as penA, are associated with a reduced affinity, and thus a decrease in susceptibility, to penicillin. All isolates in cluster A had the penA_59 allele, and isolates in cluster B had the penA_253 allele. These alleles differ by $101 \mathrm{nt}$, and the encoded peptides differ at 25 aa positions. The different amino acid residues are located in the second half of the protein, which contains the transpeptidase domain for penicillin binding. Six of the amino acid mutations encoded by penA_253 (F504L, A510V, N512Y, I515V, H541N, and I566V) have been reported to be associated with decreased susceptibility to penicillin (15).

The penA_253 allele was identified in MenB isolates of the cc32 lineage in Europe in early 2012. The PubMLST

Figure 1. Neighbor-joining dendrogram (500 bootstrap values) for core genome sequences of clonal complex 11 Neisseria meningitidis strains with serogroup W capsules, Western Australia, Australia, January 2013-December 2016. The resistance phenotype for the penicillin $G$ (PenG) gene for each isolate is provided using the following breakpoints: sensitive (MIC $\leq 0-0.06 \mathrm{mg} / \mathrm{L}$ ), intermediate $(0.12-0.25 \mathrm{mg} / \mathrm{L})$ and resistant $(\geq 0.5 \mathrm{mg} / \mathrm{L})$. Two clusters ( $\mathrm{A}$ and $\mathrm{B}$ ) were observed, which contain isolates that differ in penicillin resistance profile. Of 1,605 core-genome loci, a minimum of 244 are different between clusters $A$ and $B$. The more recent cluster $B$ appeared in early 2016 and contains penicillin-resistant isolates. Strain ExNm672 does not belong to either cluster. The dendrogram is drawn to scale, and sum of branch lengths between 2 strains indicates the proportion of nucleotide

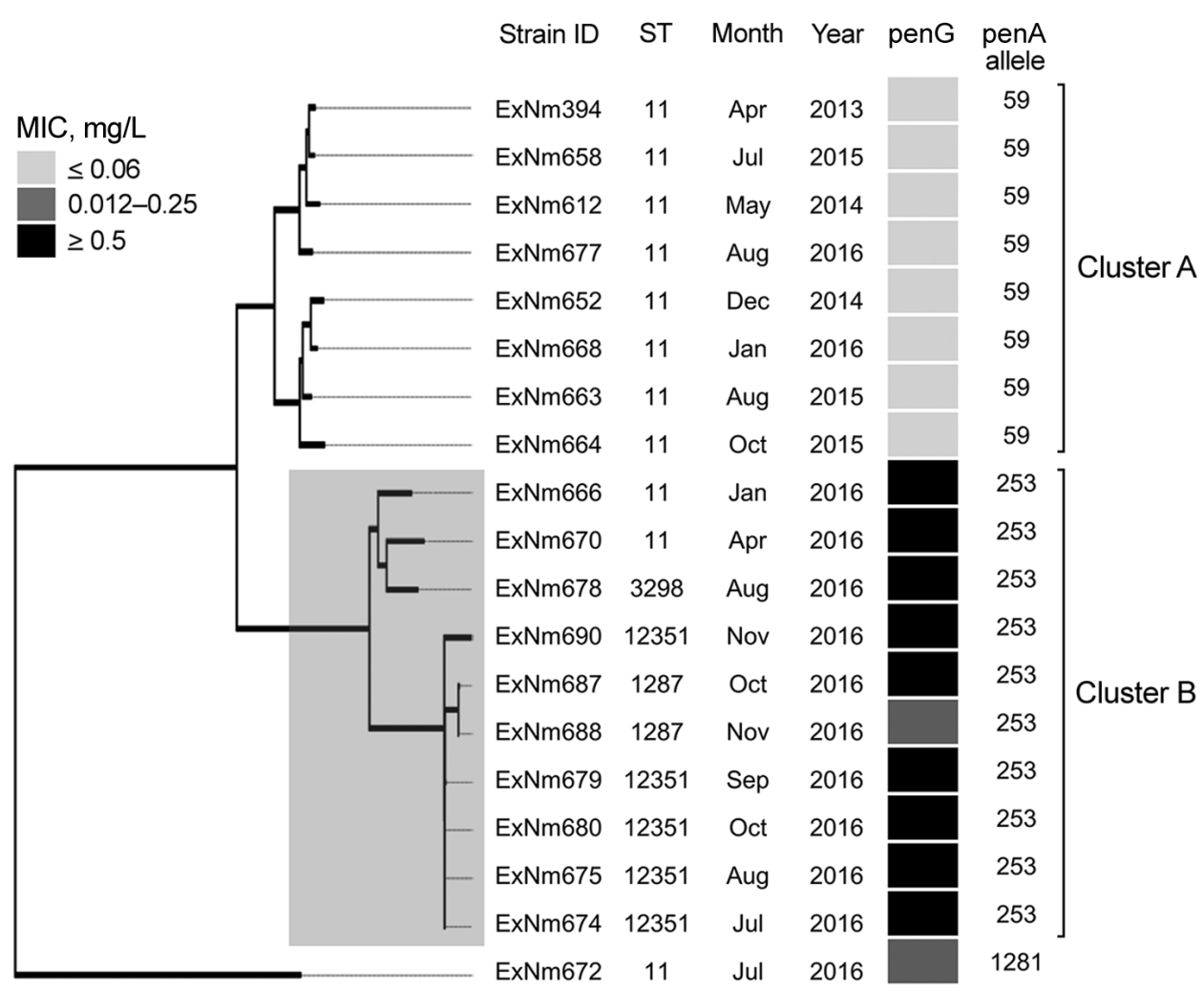
differences between those core genomes $(\approx 1.5 \mathrm{Mb})$ within the pairwise alignment. Gray shaded box indicates isolates in cluster B. Scale bar indicates nucleotide substitutions per site. ID, identification; ST, sequence type. 


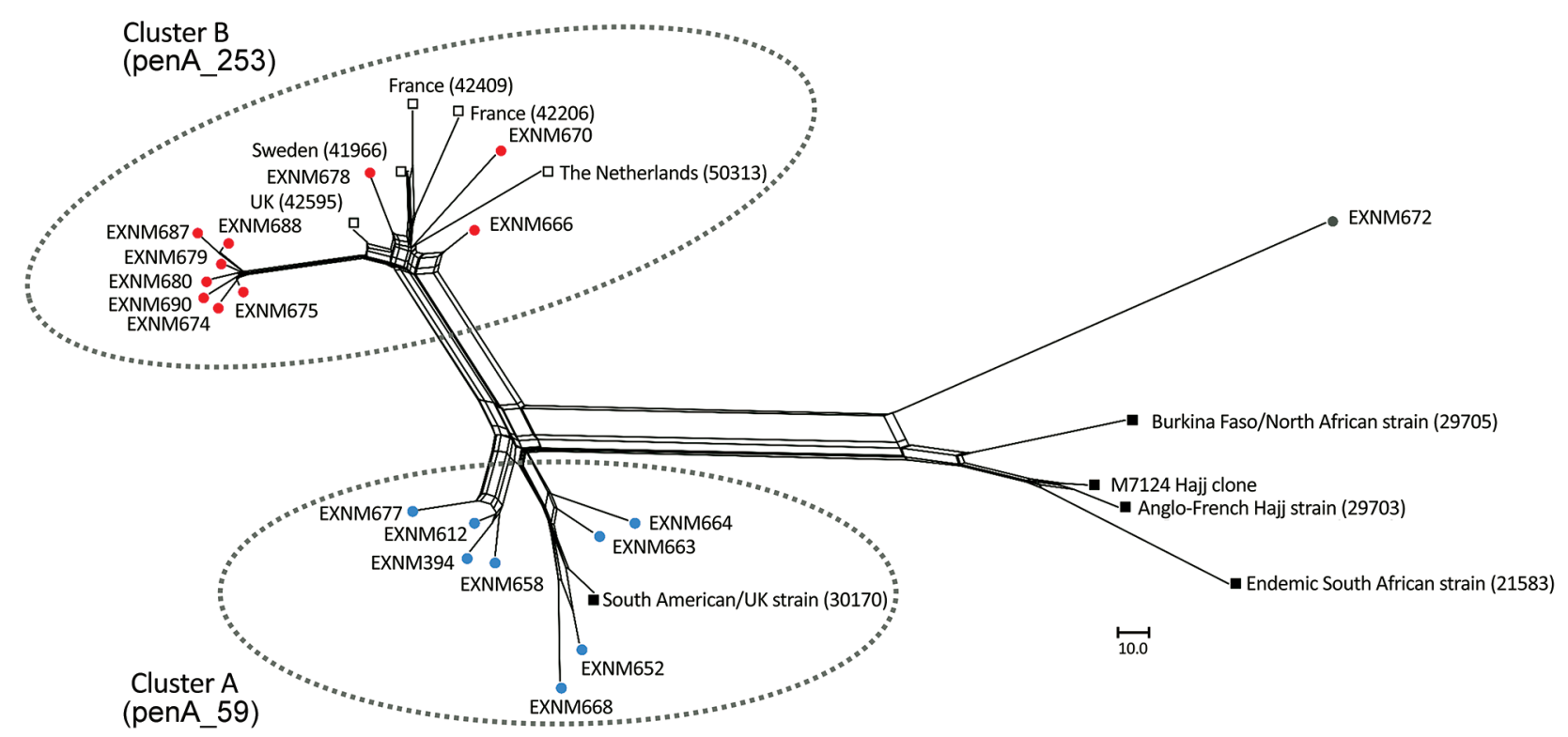

Figure 2. Phylogenetic reconstruction by using an unrooted neighbor-net algorithm of core genomes of clonal complex 11 Neisseria meningitidis strains with serogroup W capsules (MenW:cc11), Western Australia, Australia, January 2013-December 2016. Blue circles indicate isolates in cluster A from Western Australia; red circles indicate isolates in cluster B from Western Australia; gray circle indicates ExNm672, a strain isolated from a traveler; open squares indicate the $5 \mathrm{MenW}: \mathrm{cc} 11$ isolates in the PubMLST database (http://pubmlst. org/neisseria) that contains the penA_253 allele; and black squares indicate reference MenW:cc11 strains, isolated after 2010, as described by Lucidarme et al. (8). M7124 is the Hajj clone isolated in Saudi Arabia in 2000. Numbers in parentheses indicate PubMLST numbers of reference isolates. Scale bar indicates nucleotide substitutions per site.

database has 5 MenW:cc11 invasive isolates harboring this allele, all of which were obtained in Europe in 2016: 2 from France, 1 from Sweden, 1 from the United Kingdom, and 1 from the Netherlands. Core-genome analysis showed clustering of these isolates from Europe with cluster B isolates from Western Australia. (Figure 2).

To assess whether penA was responsible for the difference in penicillin resistance between the 2 clusters, the penA_253 allele from cluster B was transformed into all 8 penicillin-sensitive isolates in cluster A. We subsequently tested the penA_253 isogenic mutants obtained for penicillin resistance by using the Etest. All transformants displayed intermediate resistance to penicillin (4-fold increase in MIC to $0.25 \mathrm{mg} / \mathrm{L}$ ). These results indicate that the penA_253 allele plays a major role in increased resistance to penicillin among cluster $\mathrm{B}$ isolates.

However, exchange of penA did not fully account for the level of resistance displayed by drug-resistant clinical isolates. This finding suggests that acquisition of penicillin resistance among cluster $\mathrm{B}$ isolates is multifactorial. Because PBP1, PBP3, and PBP4 were identical in all isolates in clusters $\mathrm{A}$ and $\mathrm{B}$, there must be additional as yet undetermined factors that play a role in conferring resistance to penicillin in cluster B isolates. A comparison of the core and accessory genomes of isolates in the 2 clusters is required to further elucidate this issue.

\section{Conclusions}

MenW is now the predominant serogroup causing IMD in Western Australia. Core-genome analysis identified a new cluster of penicillin-resistant MenW:cc11 clinical isolates that emerged throughout this region during early 2016. We demonstrated that the penA_253 allele has a major role in increasing penicillin resistance among isolates in this new cluster. Because penA_253 has been identified in MenW:cc11 isolates in Europe in 2016, jurisdictions are encouraged to monitor emergence of strains harboring this allele by PCR for culture-negative cases.

\section{Acknowledgments}

We thank the scientific staff in the microbiology laboratory at PathWest Medicine, Perth, Australia, for serogrouping, storing, and collecting data for the meningococcal isolates.

This study was supported by a grant from the Amanda Young Foundation (http://www.amandayoungfoundation.org.au/), a not-for-profit, charitable organization of Western Australia, to C.M.K. C.M.K. is also supported by the National Health and Medical Research Council of Australia (APP1003697). S.M. is supported by an International Postgraduate Research Scholarship from the University of Western Australia. We used the Neisseria Multi Locus Sequence Typing website (http://pubmlst.org/ neisseria), which is supported by grant 104992 from the Wellcome Trust to Martin Maiden and K.A.J. 
Mr. Mowlaboccus is a doctoral candidate and a sessional lecturer at the University of Western Australia, Perth, Australia. His primary research interests include evolution and changing epidemiology of $N$. meningitidis and investigating the mechanism of antimicrobial resistance for this microorganism.

\section{References}

1. Maiden MC, Bygraves JA, Feil E, Morelli G, Russell JE, Urwin R, et al. Multilocus sequence typing: a portable approach to the identification of clones within populations of pathogenic microorganisms. Proc Natl Acad Sci U S A. 1998;95:3140-5. http://dx.doi.org/10.1073/pnas.95.6.3140

2. Meningitis control in countries of the African meningitis belt, 2015 . Wkly Epidemiol Rec. 2016;91:209-16.

3. Caugant DA, Maiden MC. Meningococcal carriage and diseasepopulation biology and evolution. Vaccine. 2009;27(Suppl 2):B6470. http://dx.doi.org/10.1016/j.vaccine.2009.04.061

4. Mowlaboccus S, Perkins TT, Smith H, Sloots T, Tozer S, Prempeh LJ, et al. Temporal changes in BEXSERO ${ }^{\circledR}$ antigen sequence type associated with genetic lineages of Neisseria meningitidis over a 15-year period in Western Australia. PLoS One. 2016;11:e0158315. http://dx.doi.org/10.1371/journal.pone.0158315

5. Martin NV, Ong KS, Howden BP, Lahra MM, Lambert SB, Beard FH, et al.; Communicable Diseases Network Australia MenW Working Group. Rise in invasive serogroup W meningococcal disease in Australia 2013-2015. Commun Dis Intell Q Rep. 2016;40:E454-9.

6. Carville KS, Stevens K, Sohail A, Franklin LJ, Bond KA, Brahmi A, et al. Increase in meningococcal serogroup $\mathrm{W}$ disease, Victoria, Australia, 2013-2015. Emerg Infect Dis. 2016;22:1785-7. http://dx.doi.org/10.3201/eid2210.151935

7. Ladhani SN, Beebeejaun K, Lucidarme J, Campbell H, Gray S, Kaczmarski E, et al. Increase in endemic Neisseria meningitidis capsular group $\mathrm{W}$ sequence type 11 complex associated with severe invasive disease in England and Wales. Clin Infect Dis. 2015;60:578-85. http://dx.doi.org/10.1093/cid/ciu881
8. Lucidarme J, Hill DM, Bratcher HB, Gray SJ, du Plessis M, Tsang RS, et al. Genomic resolution of an aggressive, widespread, diverse and expanding meningococcal serogroup $\mathrm{B}, \mathrm{C}$ and $\mathrm{W}$ lineage. J Infect. 2015;71:544-52. http://dx.doi.org/10.1016/ j.jinf.2015.07.007

9. Oppenheim BA. Antibiotic resistance in Neisseria meningitidis. Clin Infect Dis. 1997;24(Suppl 1):S98-101. http://dx.doi.org/ 10.1093/clinids/24.Supplement 1.S98

10. Zapun A, Morlot C, Taha MK. Resistance to $\beta$-lactams in Neisseria ssp due to chromosomally encoded penicillin-binding proteins. Antibiotics (Basel). 2016;5:E35. http://dx.doi.org/ 10.3390/antibiotics5040035

11. Turner PC, Southern KW, Spencer NJ, Pullen H. Treatment failure in meningococcal meningitis. Lancet. 1990;335:732-3. http://dx.doi.org/10.1016/0140-6736(90)90852-V

12. Jolley KA, Maiden MC. BIGSdb: scalable analysis of bacterial genome variation at the population level. BMC Bioinformatics. 2010;11:595. http://dx.doi.org/10.1186/1471-2105-11-595

13. Bond KA, Stevens K, Bulach D, Carville K, Ong KS, Howden BP. Rising incidence of invasive meningococcal disease caused by Neisseria meningitidis serogroup W in Victoria. Med J Aust. 2016;204:265-6. http://dx.doi.org/10.5694/ mja15.01222

14. Bratcher HB, Corton C, Jolley KA, Parkhill J, Maiden MC. A gene-by-gene population genomics platform: de novo assembly, annotation and genealogical analysis of 108 representative Neisseria meningitidis genomes. BMC Genomics. 2014;15:1138. http://dx.doi.org/10.1186/1471-2164-15-1138

15. Harrison OB, Clemence M, Dillard JP, Tang CM, Trees D, Grad YH, et al. Genomic analyses of Neisseria gonorrhoeae reveal an association of the gonococcal genetic island with antimicrobial resistance. J Infect. 2016;73:578-87. http://dx.doi.org/10.1016/ j.jinf.2016.08.010

Address for correspondence: Shakeel Mowlaboccus, School of Biomedical Sciences, University of Western Australia, 35 Stirling Hwy, Crawley, Perth,WA 6009, Australia; email: shakeel.mowlaboccus@uwa.edu.au

\section{EID Podcast: A Tick on the Move?}

Rickettsia parkeri, a tickborne bacterium that causes a febrile, eschar-associated illness throughout many countries of the Western Hemisphere, is transmitted by Amblyomma ticks. In the United States, more than 40 cases of $R$. parkeri rickettsiosis have been reported since its recognition in 2004. The Gulf Coast tick (Amblyomma maculatum) is the principal vector of $R$. parkeri in the United States, and all previously documented US infections arose within the known geographic range of these ticks. Confirmed cases of $R$. parkeri rickettsiosis also have been reported from Uruguay and Argentina, where $A$. triste and A. tigrinum ticks serve as the principal vector species. Recent reviews of tick collection records and archived specimens documented and identified the presence of ticks very closely related to $A$. triste in several regions of the southwestern United States and adjacent regions of Mexico since at least 1942. 